\title{
Direct and indirect effects of marijuana use on the risk of fatal 2-vehicle crash initiation
}

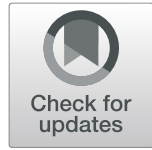

Stanford Chihuri ${ }^{1}$ and Guohua $\mathrm{Li}^{1,2^{*}}$

\begin{abstract}
Background: Marijuana and alcohol each play a significant role in fatal crash initiation. We decomposed the total effect of marijuana use in the presence or absence of alcohol on fatal crash initiation into direct and indirect effects.

Methods: Pair-matched data on 5856 culpable drivers (initiators) and 5856 nonculpable drivers (noninitiators) involved in the same fatal 2-vehicle crashes recorded in the Fatality Analysis Reporting System between 2011 and 2016 were analyzed using the conditional logistic regression model and the unified mediation and interaction analysis framework.

Results: Crash initiators were more likely than noninitiators to test positive for marijuana (16.1\% vs. 9.2\%, $P<0.001$ ), alcohol $(28.6 \%$ vs. $9.7 \%, P<0.001)$ and both substances $(6.3 \%$ vs. $1.6 \%, P<.0001)$. Adjusted odds ratios of fatal $2-$ vehicle crash initiation revealed a positive interaction on the additive scale between marijuana and alcohol. Of the total effect of marijuana use on fatal 2-vehicle crash initiation, $68.8 \%$ was attributable to the direct effect (51.5\% to controlled direct effect and $17.3 \%$ to reference interaction effect with alcohol) and $31.2 \%$ to the indirect effect (7.8\% to mediated interaction effect and $23.4 \%$ to pure indirect effect through alcohol).

Conclusion: Our results indicate that the increased odds of fatal 2-vehicle crash initiation associated with marijuana use is due mainly to the direct effect.
\end{abstract}

Keywords: Alcohol, Causal inference, Driving safety, Marijuana, Motor vehicle crashes

\section{Background}

Driving under the influence of drugs has increased over the past two decades and poses a serious threat to traffic safety in the United States (Asbridge et al. 2012; Governors Highway Safety Association (GHSA) 2018; Hartman and Huestis 2013). In 2016, 37.9\% of fatally injured drivers tested positive for alcohol, $43.6 \%$ for nonalcohol drugs, and $50.5 \%$ for two or more substances (Governors Highway Safety Association (GHSA) 2018). Marijuana is the most commonly detected nonalcohol drug and its concurrent use with alcohol is the most common

\footnotetext{
* Correspondence: GL2240@cumc.columbia.edu

'Department of Anesthesiology, Columbia University Vagelos College of Physicians and Surgeons, 622 West 168th St, PH5-505, New York, NY 10032, USA

${ }^{2}$ Department of Epidemiology, Columbia University Mailman School of Public Health, 622 West 168th St, PH5-505, New York, NY 10032, USA
}

polydrug combination among drivers (Berning et al. 2015; Bonar et al. 2018; Governors Highway Safety Association (GHSA) 2018). Although the prevalence of alcohol-impaired driving has declined in recent years in the United States, it still contributes to $28 \%$ of all trafficinjury fatalities or 29 deaths daily (National Center for Statistics and Analysis (NCSA) 2017; National Center for Statistics and Analysis (NCSA) 2019a). About one quarter of fatally injured drivers test positive for marijuana (Governors Highway Safety Association (GHSA) 2018). In 2017, 22.1\% of adults aged 18 to 25 years reported use of marijuana in the previous month and $11.3 \%$ reported driving under the influence of drugs in the past year (Center for Behavioral Health Statistics and Quality (CBHSQ) 2018; Substance Abuse and Mental Health Services Administration (SAMHSA) 2018). Each year, about one million people are arrested for 
driving under the influence of drugs in the United States (Federal Bureau of Investigations (FBI) 2019). This number is expected to increase as marijuana becomes more permissible and accessible.

As of November 15, 2019, 34 states and the District of Columbia have enacted medical marijuana laws while 11 states and the District of Columbia have legalized recreational marijuana (National Conference of State Legislatures (NCSL) 2019a; National Conference of State Legislatures (NCSL) 2019b). Owing to its putative analgesic effects, state governments are increasingly considering marijuana as a viable alternative to prescription opioids in chronic pain management (Chihuri and $\mathrm{Li}$ 2019; National Academies of Sciences, Engineering, and Medicine (NASEM) 2017). For example, in Colorado, New York, and Illinois, individuals with opioid prescriptions or certain health conditions can now legally purchase medical marijuana at a registered dispensary (Quinton 2019). As more states consider legalizing medical and recreational marijuana, it is important to understand the health consequences of marijuana use, such as its effect on driving safety. Currently, 12 states have zero tolerance laws that prohibit driving with any amount of marijuana in the body, 5 states have per se laws that prohibit driving with marijuana in excess of the legal limit, and 1 state has a permissible inference law that permits law enforcement to assume driving under the influence if delta-9-tetrahydrocannabinol (THC) exceeds the allowable threshold (NCSL 2019c). All other states have laws prohibiting driving under the influence of marijuana based on field sobriety tests and observation by law enforcement officers (Wong et al. 2014; Governors Highway Safety Association (GHSA) 2018; NCSL 2019c).

Use of marijuana can slow reaction time, impair judgement and concentration, and decrease psychomotor skills (Downey et al. 2013; Hartman and Huestis 2013; Hartman et al. 2015; Lenne et al. 2010; Lipari et al. 2016; Robbe 1998; Rogeberg and Elvik 2016). Previous epidemiological studies have found a positive interaction on the additive scale between marijuana and alcohol on fatal crash involvement and initiation (Chihuri et al. 2017; Drummer et al. 2004; Dubois et al. 2015; Gjerde et al. 2011; Laumon et al. 2005; Li et al. 2017; Lipari et al. 2016; Rogeberg and Elvik 2016). Experimental studies have also reported additivity at high concentrations of THC and alcohol (Ramaekers et al. 2004; Robbe 1998; Sewell et al. 2009). However, little is known about the causal pathways linking the concurrent use of marijuana and alcohol to increased risks of crash involvement and initiation. Previous studies of polydrug use and driving safety have assessed interaction but not mediation. The traditional approach to mediation analysis is known to have limitations and be susceptible to bias resulting from exposure-mediator interaction (Richiardi et al. 2013). However, recent development in epidemiologic methods has made it possible to simultaneously assess mediation and interaction (Bellavia and Valeri 2018; VanderWeele 2014; Wang and Arah 2015). The counterfactual framework allows for decomposition of the total effect into direct and indirect effects: hence, disentangling the different pathways linking exposure to outcome (Richiardi et al. 2013; VanderWeele 2014). The present study aims to quantify the direct and indirect effects of marijuana use on the risk of fatal 2-vehicle crash initiation through the unified framework for interaction and mediation analysis (VanderWeele 2014). The unified framework allows for further partitioning the direct effect into controlled direct effect and reference interaction effect, and the indirect effect into mediated interaction effect and pure indirect effect.

\section{Methods}

\section{Data source}

Data for this study came from the Fatality Analysis Reporting System (FARS), which is maintained by the National Center for Statistics and Analysis of the National Highway Traffic Safety Board. Since 1975, the FARS has served as the census of fatal motor vehicle crashes occurring on public roads in the United States. Crashes eligible to be recorded in the FARS are those that have resulted in at least one personal fatality (i.e., a driver, passenger, or a non-occupant) within 30 days of the crash (National Highway Traffic Safety Administration (NHTSA) 2018; Wang and Arah 2015). FARS data are collected from various sources, including death certificates, coroner/medical examiner reports, police crash reports, state vehicle registration files, state driver licensing files, emergency medical service reports, and vital statistics (National Center for Statistics and Analysis (NCSA) 2019b; National Highway Traffic Safety Administration (NHTSA) 2018). This study was deemed not human subjects research Under 45 CFR 46 by the Columbia University Intitutional Review Board (New York, NY).

Trained FARS analysts use standardized operational manuals and uniform coding practices to code more than 140 de-identified data elements into as many as 20 data files each year National Center for Statistics and Analysis (NCSA) 2019b). Data files relevant to this study include the accident, vehicle, and person files. The accident file contains environmental and crash circumstances (e.g., road and weather conditions), the vehicle file contains characteristics of the involved vehicles (e.g., make, model, and body type), and the person file includes demographic and other characteristics for each involved person (e.g., driver age, sex, driving history, and drug testing results) National Center for Statistics and Analysis (NCSA) 2019b; National Highway Traffic Safety 
Administration (NHTSA) 2018). Quality assurance programs automatically check the data for completeness, timeliness, consistency, and accuracy (National Center for Statistics and Analysis (NCSA) 2019b).

In this study, driver-related factors or unsafe driver actions such as lane weaving or speeding (codes 17-60), obtained from police reports and other supporting documents, were used to assign crash responsibility (National Highway Traffic Safety Administration (NHTSA) 2018). For each crash, up to 4 unsafe driver actions were recorded (National Highway Traffic Safety Administration (NHTSA) 2018). Most unsafe driver actions or errors are considered to have contributed to the crash (Blower 1998). In this study, the driver with one or more unsafe driver actions or errors was regarded as the crash initiator, while the other driver without any errors was regarded as the noninitiator. Driving errors are commonly used as a proxy for culpability. Compared to traffic violations that may require legal proof (Blower 1998), driving errors tend to be uniformly applied and to fit the configuration of the crash site, i.e., vehicle positioning, skid marks, and severity of structural damage. Assignment of driving errors is based on the configuration and evidence on the crash scene as well as interviews with witnesses (Blower 1998). Two-vehicle crashes where both drivers made at least one driving error (i.e., shared culpability) were excluded from this study.

\section{Study design and population}

A pair-matched study design was used to assess the individual and joint effects of marijuana and alcohol on the risk of fatal 2-vehicle crash initiation. In this pairmatched study, crash initiators were drivers who were responsible for initiating the fatal 2-vehicle crashes while noninitiators were drivers who were involved in the same 2-vehicle crashes but were not responsible for these crashes. From January 2011 to December 2016, the FARS recorded a total 187,870 fatal crashes involving 280,041 drivers. Excluded from the analysis were 112, 643 crashes involving a single vehicle or more than 2

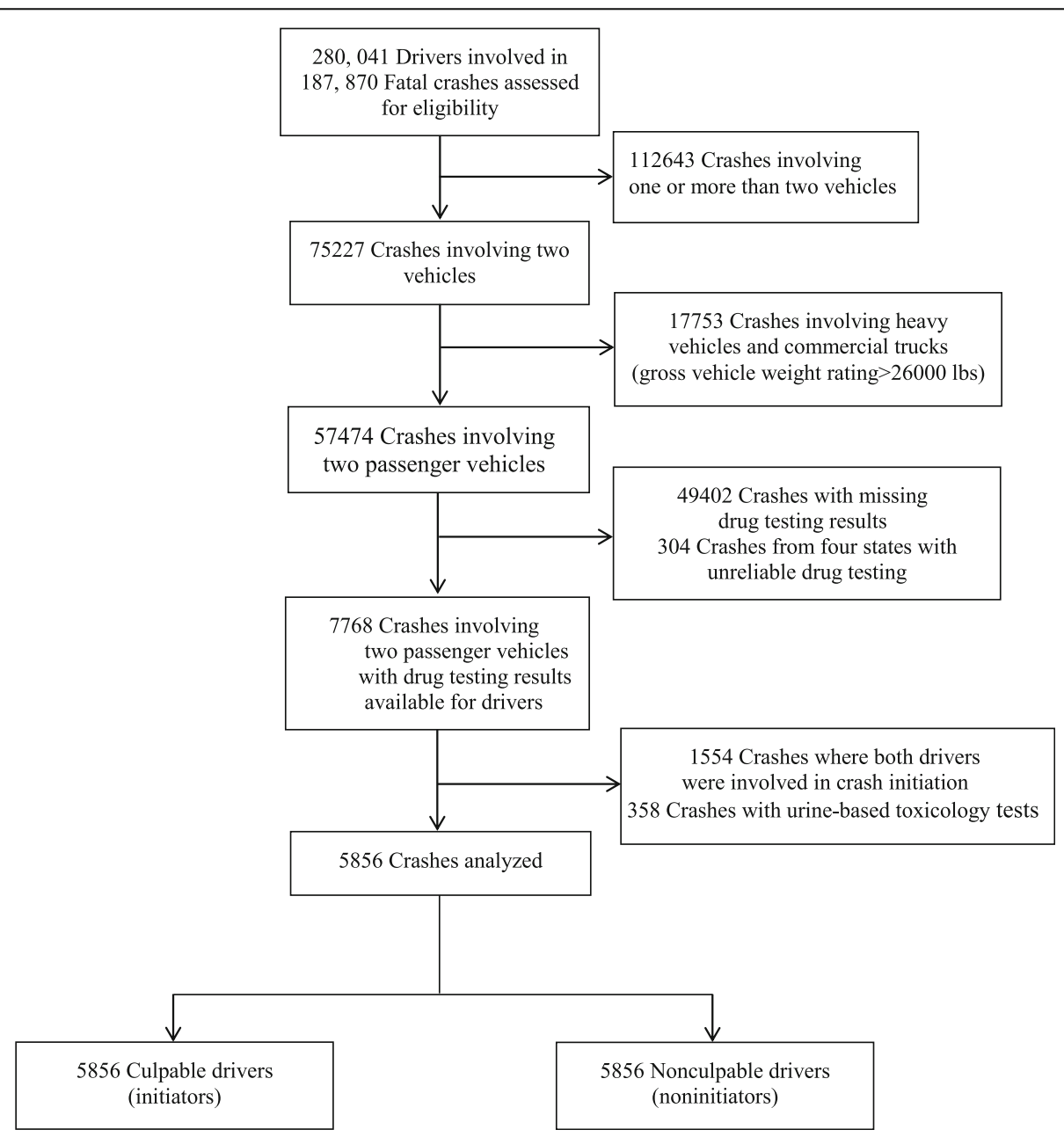

Fig. 1 Selection of drivers involved in fatal 2-vehicle crashes, Fatality Analysis Reporting System, 2011-2016 
vehicles, 17,753 crashes involving heavy vehicles or commercial vehicles (gross vehicle weight rating 26,000 lbs.), 49,402 crashes with missing toxicological testing results, 304 crashes in Maryland, Montana, New Mexico, and North Carolina with toxicological testing results recorded unreliably in the FARS, 1554 2-vehicle crashes in which both drivers were culpable of crash initiation, and 358 2-vehicle crashes where toxicology tests were based on urine samples (Fig. 1). Included in the study were 5856 pairs of drivers involved in 5856 fatal 2-vehicle crashes with complete toxicological testing data.

\section{Drug testing assessments}

Injury fatalities from motor vehicle crashes are usually investigated by medical examiners or coroners (Executive Office of the President, National Science and Technology Council (EOP-NSTC) 2016). In the United States, 26 states and the District of Columbia have centralized or state medical examiner systems, 12 have coroner systems, and 12 have a county-based systems with a mixture of coroner and medical examiner offices (Centers for Disease Control and Prevention (CDC) 2015; Davis et al. 2015). Overall, approximately 2400 medicalexaminer and coroner jurisdictions are responsible for conducting autopsies and performing toxicological tests across the United States (Executive Office of the President, National Science and Technology Council (EOPNSTC) 2016). For nonfatally injured drivers involved in fatal crashes, blood samples are usually taken at the medical facility where they are treated and those blood samples at admission are routinely used for toxicological analysis (Li et al. 2011).

Toxicological drug tests were conducted on blood or urine specimens using liquid/gas chromatography and radioimmunoassay techniques for screening, and liquid/ gas chromatography and mass spectrometry for confirmation (Kaplan et al. 2006; Li et al. 2011). All drivers included in this study had at least one toxicological drug test based on a blood specimen. Prior to 2018, the FARS recorded up to 3 nonalcohol drugs. In instances where a drug metabolite was detected, only the parent drug was recorded National Center for Statistics and Analysis (NCSA) 2019b; National Highway Traffic Safety Administration (NHTSA) 2018). If more than 3 nonalcohol drugs were detected, the FARS recorded the drugs in the following priority: narcotics, depressants, stimulants, marijuana, and other drugs (Kaplan et al. 2006; National Highway Traffic Safety Administration (NHTSA) 2018). In the present study, marijuana refers to cannabinoids such as THC and/or other cannabinoid metabolites (codes 600-695) (National Center for Statistics and Analysis (NCSA) 2019b). Blood alcohol concentrations (BACs) were measured and recorded separately from nonalcohol drugs and a BAC of $0.01 \mathrm{~g} / \mathrm{dL}$ or greater was considered alcohol-positive (National Center for Statistics and Analysis (NCSA) 2019b; National Highway Traffic Safety Administration (NHTSA) 2018). We also analyzed BAC data as a 3-level categorical variable (BACs $<0.01,0.01-0.07$, and $\geq 0.08 \mathrm{~g} / \mathrm{dL}$ ).

\section{Statistical analysis}

Frequency distributions of driver characteristics were tabulated by crash initiation status. The McNemar's test was used to compare initiators and noninitiators on driver characteristics such as age, sex, marijuana testing result, alcohol testing result, driving history within the previous 3 years, and survival status. The Pearson $x^{2}$ test was use to compare initiators and noninitiators on age categories and BAC levels. The Cochran Armitage trend test was used to assess the changes in the prevalence of marijuana detected in drivers over the study period. Conditional logistic regression modeling was used to estimate crude and adjusted odds ratios (ORs) and 95\% confidence intervals (CIs) of crash initiation associated with marijuana use, alcohol use, and other driver characteristics. To assess separate and joint effects of marijuana and alcohol, drivers testing negative for marijuana and alcohol were assigned as the reference group. The interaction of marijuana and alcohol was assessed on the multiplicative and additive scales. Additive interaction was assessed using 3 statistics: the relative excess risk due to interaction (RERI), attributable proportion due to interaction (AP), and the synergy index (S). The corresponding 95\% CIs were computed using a method suggested by Zou (2008).

The unified framework for interaction and mediation analysis developed by VanderWeele (2014) was used to quantify the direct and indirect effects of marijuana use on the risk of crash initiation (Fig. 2). The total effect of marijuana was decomposed into 4 components: 1 ) controlled direct effect, which refers to the effect of marijuana on the risk of crash initiation in the absence of alcohol (i.e., the portion of the total effect of marijuana that is due to neither interaction nor mediation); 2) reference interaction, which refers to the combined effect of marijuana and alcohol on the risk of crash initiation if alcohol is not in itself necessary for crash initiation from marijuana use (i.e., the portion of the total effect of marijuana that is due to interaction only); 3) mediated interaction, which refers to the combined effect of marijuana and alcohol on the risk of crash initiation if alcohol is necessary for crash initiation from marijuana use (i.e., the portion of the total effect of marijuana that is due to both mediation and interaction); and 4) pure indirect effect, which refers to the effect of marijuana on the risk of crash initiation operated through alcohol as the mediator (i.e., the portion of the 


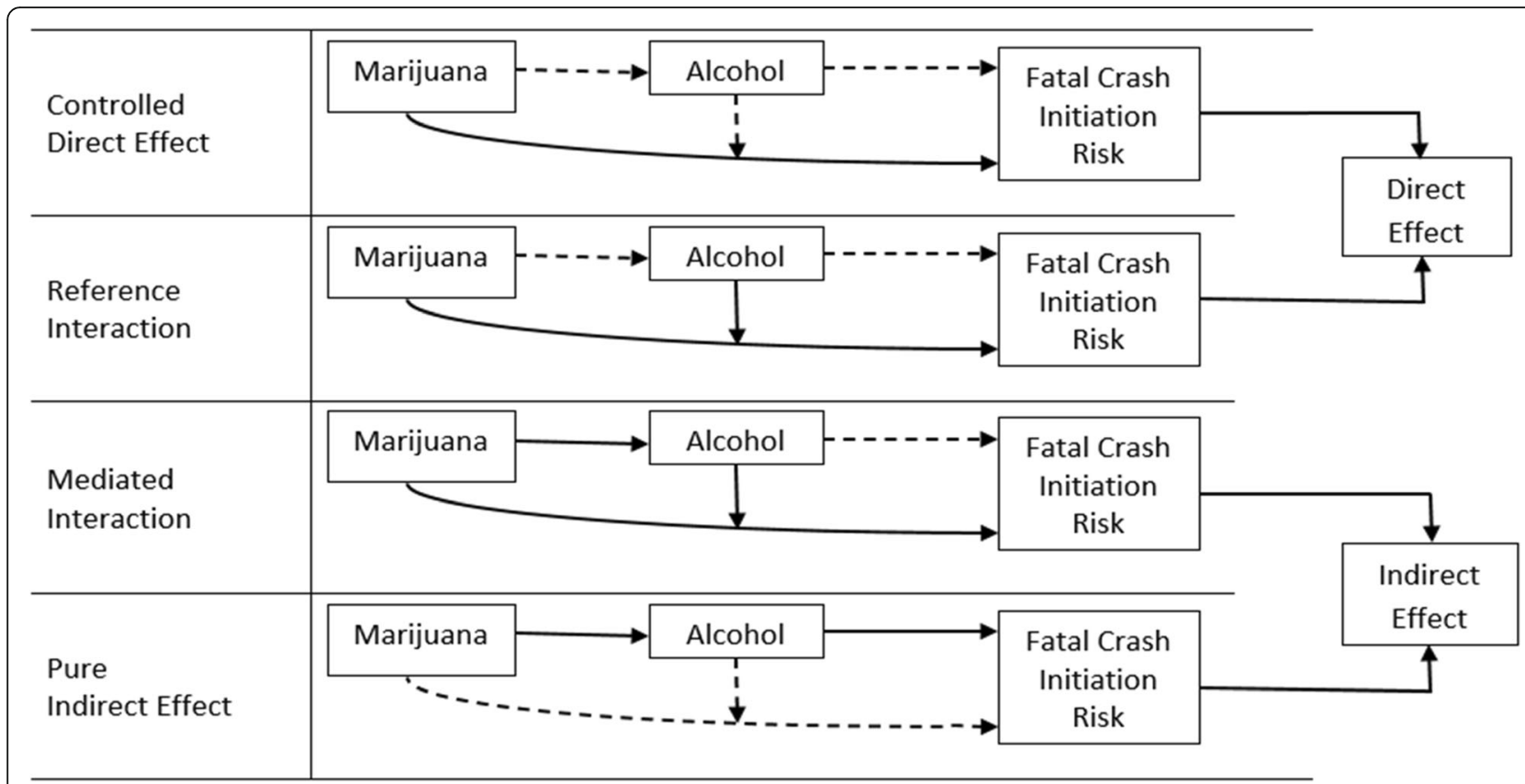

Fig. 2 Graphical explanation of the four component effects of marijuana use on fatal 2-vehicle crash initiation in the presence of alcohol under the unified framework for mediation and interaction analysis proposed by WanderWeele (2014)

total effect of marijuana that is due to mediation through alcohol only). The direct effect comprises the controlled direct effect and reference interaction whereas the indirect effect is made up of the mediated interaction and pure indirect effect (Fig. 2). All data analyses were performed using SAS, version 9.4 (SAS
Institute Inc., Cary, NC). Statistical significance was set at $P<0.05$ for 2 -tailed tests.

\section{Results}

Compared with drivers excluded from the study due to missing or incomplete drug testing results, those

Table 1 Frequency distribution of driving errors involved in 5856 fatal 2-vehicle crashes by marijuana positivity status, Fatality Analysis Reporting System, 2011-2016

\begin{tabular}{|c|c|c|c|}
\hline Type of driving error & $\begin{array}{l}\text { Positive for } \\
\text { marijuana } \\
\text { No. (\%) }\end{array}$ & $\begin{array}{l}\text { Negative for } \\
\text { marijuana } \\
\text { No. (\%) }\end{array}$ & $\begin{array}{l}\text { Total } \\
\text { No. }(\%)\end{array}$ \\
\hline Failure to keep in proper lane & $322(22.6)$ & $1577(25.3)$ & $\begin{array}{l}1899 \\
(24.8)\end{array}$ \\
\hline Failure to yield right of way & $148(10.4)$ & $1299(20.8)$ & $\begin{array}{l}1447 \\
(18.9)\end{array}$ \\
\hline Driving too fast for conditions or in excess of posted maximum & $320(22.4)$ & $1037(16.6)$ & $\begin{array}{l}1357 \\
(17.7)\end{array}$ \\
\hline Failure to obey actual traffic signs, traffic control devices, or traffic officers & $150(10.5)$ & $684(11.0)$ & $834(10.9)$ \\
\hline $\begin{array}{l}\text { Operating the vehicle in an erratic, reckless, careless, or negligent manner or at erratic or } \\
\text { suddenly changing speeds }\end{array}$ & $110(7.7)$ & $339(5.4)$ & $449(5.9)$ \\
\hline Driving on wrong side of the road & $107(7.5)$ & $541(8.7)$ & $648(8.4)$ \\
\hline Manslaughter or homicide or other assault & $93(6.5)$ & $313(5.0)$ & $406(5.3)$ \\
\hline Making an improper turn & $24(1.7)$ & $132(2.1)$ & $156(2.0)$ \\
\hline Passing with insufficient distance or inadequate visibility or failing to yield to overtaking vehicle & $31(2.2)$ & $94(1.5)$ & $125(1.6)$ \\
\hline Passing where prohibited & $60(4.2)$ & $16(0.3)$ & $76(1.0)$ \\
\hline Any other & $62(4.3)$ & $211(3.4)$ & $273(3.6)$ \\
\hline Total $^{\mathrm{a}}$ & $1427(100.0)$ & $6243(100.0)$ & $\begin{array}{l}7670 \\
(100.0)\end{array}$ \\
\hline
\end{tabular}

${ }^{a}$ Total exceeds the number of crashes because more than 1 error could be recorded in each crash 
included in the analysis were slightly younger (mean age: 42.2 years, standard deviation:18.3 years vs. mean age: 43.7 years, standard deviation: 19.1 years, $P<0.0001$ ), more likely to be male $(71.3 \%$ vs. $70.3 \%, P=0.028)$, and more likely to be involved in a crash in the previous 3 years $(23.1 \%$ vs. $22.2 \%, P=0.026)$. Drivers included in the analysis did not significantly differ from the excluded drivers with regards to survival status and other driving histories in the previous 3 years, such as license suspension, driving-while-intoxicated conviction, and speeding conviction.

The most common driving error leading to fatal 2vehicle crashes was failure to keep in proper lane (24.8\%), followed by failure to yield right of way $(18.9 \%)$ (Table 1). Of the driving errors committed by drivers testing positive for marijuana, $22.6 \%$ were failure to keep in proper lane and $22.4 \%$ by speeding (Table 1 ).

Between 2011 and 2016, the prevalence of marijuana increased from 14.0 to $18.1 \%(P=0.0001)$ among crash initiators, and from 7.0 to $13.4 \%(P<0.0001)$ among noninitiators. Overall, crash initiators were more likely than noninitiators to test positive for marijuana (16.1\% vs. $9.2 \%, P<0.001)$, alcohol $(28.6 \%$ vs. $9.7 \%, P<0.001)$, and both substances $(6.3 \%$ vs. $1.6 \%, P<0.0001)$. Initiators were more likely than noninitiators to be under 35 years of age ( $50.8 \%$ vs. $33.8 \%, P<0.0001)$, to have died in the crash $(55.5 \%$ vs. $41.9 \%, P<0.0001)$, and to have had a crash $(23.0 \%$ vs. $19.2 \%, P<0.0001)$, a driving-whileintoxicated conviction $(5.8 \%$ vs. $2.8 \%, P=0.0009)$, a speeding conviction $(21.4 \%$ vs. $17.1 \%, P<0.0001)$, or license suspension $(18.7 \%$ vs. $10.0 \%, P<0.0001)$ within the previous 3 years (Table 2).

Marijuana use and alcohol use were each associated with a significantly increased risk of fatal 2-vehicle crash initiation when adjusting for driver age, sex, and driving history within the previous 3 years (Table 3 ). Compared to drivers who tested negative for both alcohol and marijuana, the estimated odds of fatal crash initiation increased 1.5-fold for those testing positive for marijuana and negative for alcohol, 5 -fold for those testing negative for marijuana and positive for alcohol, and 6.8-fold for those testing positive for both marijuana and marijuana (Table 3). The odds of crash initiation increased with BACs regardless of marijuana positivity status (Fig. 3).

There was no significant interaction on the multiplicative scale as shown by the interaction term of marijuana and alcohol $(\beta=-0.1103, P=0.4982)$. However, there was a significant interaction on the additive scale as assessed by all 3 statistics: RERI $=1.29$ (95\% CI: 0.40, 3.44), AP $=0.19$ (95\% CI: 0.09, 0.38), and $\mathrm{S}=1.29$ (95\% CI: $1.02,1.81)$. Of the total effect of marijuana on fatal crash initiation risk, $68.8 \%$ was attributed to direct effect $(51.5 \%$ to controlled direct effect and $17.3 \%$ to reference interaction with alcohol) and $31.2 \%$ to indirect effect $[7.8 \%$ to mediated
Table 2 Characteristics of drivers involved in fatal 2-vehicle crashes by crash initiation status, Fatality Analysis Reporting System, 2011-2016

\begin{tabular}{|c|c|c|c|}
\hline Driver characteristic & $\begin{array}{l}\text { Initiators }^{a} \\
n=5856 \\
N(\%)\end{array}$ & $\begin{array}{l}\text { Noninitiators }^{\mathrm{b}} \\
n=5856 \\
\mathrm{~N}(\%)\end{array}$ & $P$ value \\
\hline \multicolumn{4}{|l|}{ Age, years } \\
\hline $16-24$ & $1517(25.9)$ & $899(15.4)$ & \multirow[t]{6}{*}{$<0.0001$} \\
\hline $25-34$ & $1457(24.9)$ & $1078(18.4)$ & \\
\hline $35-44$ & $843(14.4)$ & $981(16.8)$ & \\
\hline $45-54$ & $713(12.2)$ & 1085 (18.6) & \\
\hline $55-64$ & $549(9.4)$ & $985(16.8)$ & \\
\hline$\geq 65$ & $775(13.2)$ & $822(14.1)$ & \\
\hline \multicolumn{4}{|l|}{ Sex } \\
\hline Female & $1679(28.7)$ & $1723(29.4)$ & \multirow[t]{2}{*}{0.4268} \\
\hline Male & $4175(71.3)$ & $4133(70.6)$ & \\
\hline \multicolumn{4}{|c|}{ Crash in the past 3 years } \\
\hline Yes & $1309(23.0)$ & $1098(19.2)$ & \multirow[t]{2}{*}{$<0.0001$} \\
\hline No & $4385(77.0)$ & $4606(80.8)$ & \\
\hline \multicolumn{4}{|c|}{ DWI conviction in the past 3 years } \\
\hline Yes & $338(5.8)$ & $163(2.8)$ & \multirow[t]{2}{*}{0.0009} \\
\hline No & $5494(94.2)$ & $5677(97.2)$ & \\
\hline \multicolumn{4}{|c|}{ Speeding conviction in the past 3 years } \\
\hline Yes & $1250(21.4)$ & $1000(17.1)$ & \multirow[t]{2}{*}{$<0.0001$} \\
\hline No & $4582(78.6)$ & $4840(82.9)$ & \\
\hline \multicolumn{4}{|c|}{ License suspension in the past 3 years } \\
\hline Yes & $1090(18.7)$ & $582(10.0)$ & \multirow[t]{2}{*}{$<0.0001$} \\
\hline No & $4742(81.3)$ & $5258(90.0)$ & \\
\hline \multicolumn{4}{|c|}{ Tested positive for marijuana } \\
\hline Yes & $942(16.1)$ & $541(9.2)$ & \multirow[t]{2}{*}{$<0.0001$} \\
\hline No & $4914(83.9)$ & $5315(90.8)$ & \\
\hline \multicolumn{4}{|c|}{ Tested positive for alcohol } \\
\hline$\geq 0.01$ & $1673(28.6)$ & $569(9.7)$ & \multirow[t]{2}{*}{$<0.0001$} \\
\hline 0.00 & $4183(71.4)$ & $5287(90.3)$ & \\
\hline \multicolumn{4}{|l|}{ Survival status } \\
\hline Dead & $3253(55.5)$ & $2456(41.9)$ & \multirow[t]{2}{*}{$<0.0001$} \\
\hline Alive & $2603(44.5)$ & $3400(58.1)$ & \\
\hline
\end{tabular}

Abbreviation: DWI Driving while intoxicated

${ }^{a}$ Among initiators 2 had missing data on age, 2 on sex, 24 on speeding conviction, 24 on license suspension, 24 on DWI conviction, and 162 on crash within the past 3 years

${ }^{b}$ Among noninitiators, 2 had missing data on age, 16 on speeding conviction, 16 on license suspension, 16 on DWI conviction, and 152 on crash within the past 3 years

interaction through alcohol and $23.4 \%$ to pure indirect effect (Table 4)].

\section{Discussion}

Results of this study indicate that marijuana use and alcohol use are each associated with a significantly 
Table 3 Estimated odds ratios (ORs) and 95\% confidence intervals (Cls) of fatal 2-vehicle crash initiation by driver marijuana and alcohol positivity status, Fatality Analysis Reporting System, 2011-2016

\begin{tabular}{lllllll}
\hline Marijuana & Alcohol & \multicolumn{2}{c}{ Crude } & & \multicolumn{2}{c}{ Adjusted $^{\mathrm{a}}$} \\
& & OR & $95 \% \mathrm{Cl}$ & & OR & $95 \% \mathrm{Cl}$ \\
\hline Negative & Negative & 1.00 & reference & & 1.00 & reference \\
Positive & Negative & 1.81 & $1.58,2.09$ & & 1.53 & $1.31,1.77$ \\
Negative & Positive & 4.70 & $4.11,5.39$ & & 4.95 & $4.28,5.72$ \\
Positive & Positive & 7.46 & $5.72,9.72$ & & 6.76 & $5.12,8.94$ \\
\hline
\end{tabular}

Abbreviations: OR Odds ratio, $\mathrm{Cl}$ Confidence interval

${ }^{a}$ Adjusted for age, sex, and previous driving history within the past 3 years (i.e., crash, license suspension, driving while impaired conviction, and speeding conviction)

increased risk of fatal 2-vehicle crash initiation and that concurrent use of marijuana and alcohol confers a significant interaction effect on the risk of fatal crash initiation on the additive scale. Furthermore, over two-thirds of the total effect of marijuana use on fatal crash initiation are attributed to the direct effect, including 52\% due to controlled direct effect and $17 \%$ due to reference interaction. Our findings shed more light on the causal role of marijuana use in crash initiation. Specifically, the decomposition analysis indicates that the increased risk of crash initiation associated with marijuana use is operationalized through dual pathways, with $69 \%$ being attributed to the direct effect and $31 \%$ to the indirect effect. These results are generally consistent with findings from experimental studies (Crancer Jr et al. 1969;
Moskowitz et al. 1976; Ramaekers 2003; Smiley et al. 1981) and epidemiologic studies (Asbridge et al. 2012; Li et al. 2012; Rogeberg and Elvik 2016).

There is a paucity of research assessing the mediation effect of marijuana by alcohol on crash risk and crash initiation. Our study reveals that about $23 \%$ of the total effect of marijuana use on fatal 2-vehicle initiation is due to mediation through alcohol, which is consistent with the additive effect model of risk factors where one exposure contributes to another exposure that cumulatively increase the risk of the outcome (Bean et al. 2019). Since the risk of crash initiation increases with BACs, the magnitude of the mediation effect of marijuana by alcohol is likely to increase with BACs in a doseresponse fashion. Results of the 4-way decomposition also show that $25 \%$ of the total effect was attributed to interaction (i.e., mediated interaction and reference interaction). Although the individual components of mediated interaction and reference interaction are not statistically significant, the overall interaction effect on the additive scale is statistically significant.

Assessing interactions between alcohol and other drugs on driving safety may help identify subgroups of drivers to maximize public health impact in resource allocation and risk reduction (Blot and Day 1979; Luedtke and Van der Laan 2016; Rothman et al. 1980, 2008; Saracci 1980; VanderWeele 2015). However, the relative effects across subgroups may change depending on the scale (i.e., multiplicative vs. additive). The "interaction continuum" proposed by VanderWeele (2019) provides

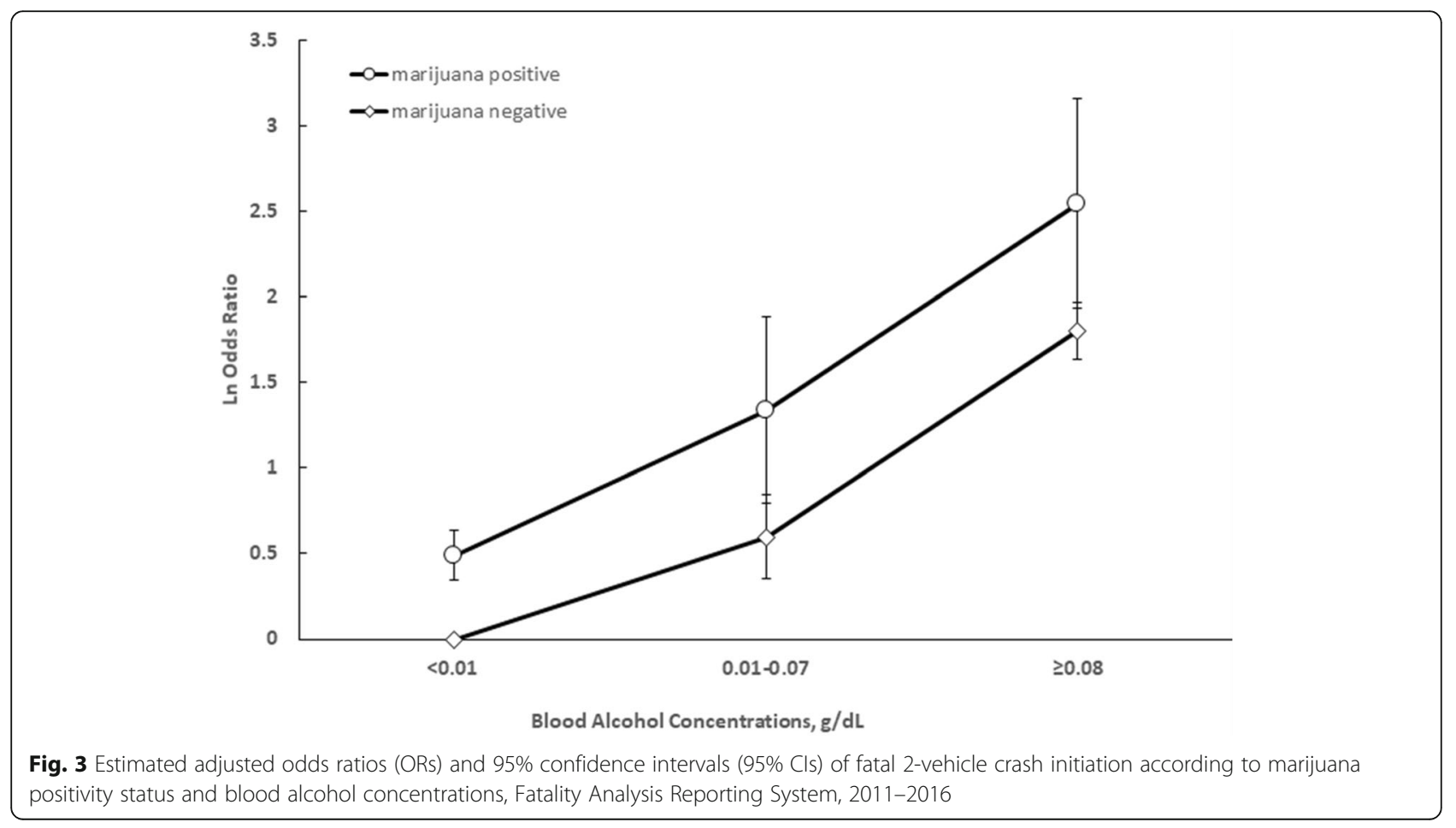


Table 4 Four-way decomposition of the total effect of marijuana use on fatal 2-vehicle crash initiation in the presence of alcohol

\begin{tabular}{|c|c|c|c|c|}
\hline Component & Log (Odds Ratio) & 95\% Confidence Interval & Effect Proportion & $P$ value \\
\hline Total Effect & 0.499 & $0.31,0.66$ & 1.00 & $<0.001$ \\
\hline Controlled Direct Effect & 0.257 & $0.16,0.36$ & 0.515 & $<0.001$ \\
\hline Reference Interaction & 0.086 & $-0.04,0.21$ & 0.173 & 0.181 \\
\hline Mediated Interaction & 0.039 & $-0.02,0.10$ & 0.078 & 0.205 \\
\hline Pure Indirect Effect & 0.117 & $0.05,0.18$ & 0.234 & 0.001 \\
\hline
\end{tabular}

an ordinal metric for gauging the strength of interaction across scales, from the strongest (positive-multiplicative positive-additive) to the weakest (inverted interaction) (VanderWeele 2019). Results from this study suggest that concurrent use of marijuana and alcohol confers an effect on fatal crash initiation that corresponds to the second strongest form of interaction on the interaction continuum, namely no-multiplicative positive additive (VanderWeele 2019).

According to VanderWeele (2014), the unified framework for mediation and interaction analysis is based on four assumptions: 1) the effect of marijuana use on crash initiation is unconfounded conditional on baseline covariates; 2) the effect of alcohol use on crash initiation is unconfounded conditional on baseline covariates and marijuana use; 3) the effect of marijuana use on alcohol use is unconfounded conditional on covariates; and 4) none of the confounders of alcohol use on crash initiation are affected by marijuana use. Although it is difficult to rigorously evaluate each of the assumptions, our study takes into consideration these assumptions through design and analytical approaches. First, the pairmatched design ensures that crash initiators and noninitiators are matched on weather, road and traffic conditions, location and time of the crash, regulation, toxicological testing protocol, and other spatiotemporal and environmental factors. Second, the conditional logistic regression model controls for driver age, sex, and driving history in the previous 3 years (i.e., speeding convictions, DWI, license suspension, and crashes). Finally, assignment of crash initiation status was based on driving errors because they do not require legal proof and they tend to be uniformly applied (Blower 1998) and have been widely used in previous culpability studies.

Our study shows that the prevalence of marijuana detected in drivers involved in fatal 2-vehicle crashes has increased steadily in the past decade. This is likely due in a large part to the increased permissibility and availability as more states have enacted laws to legalize marijuana for medical and recreational use. Marijuana use is associated with impairment of psychomotor skills necessary to operate a motor vehicle safely such as reaction time which may lead to failure to yield right of way (Chihuri et al. 2017; Downey et al. 2013; Hartman and
Huestis 2013; Lenne et al. 2010; Lipari et al. 2016; Robbe 1998; Rogeberg and Elvik 2016). Marijuana use may also impair higher-level driving skills such as hazard perception, risk management and selfcontrol, which may lead to failure in lane tracking and other driving errors (Downey et al. 2013; Hartman and Huestis 2013; Lenne et al. 2010; Lipari et al. 2016; Robbe 1998; Rogeberg and Elvik 2016). In the present study, failure to keep in proper lane and failure to yield right of way were the two most frequently identified driving errors leading to fatal 2vehicle crashes. Although alcohol use remains a much stronger risk factor for crash initiation, marijuana is associated with elevated risk both in the presence or absence of alcohol through direct and indirect pathways. As such, policymakers should consider developing countermeasures that target use of specific substances as well as polysubstance use, such as concurrent use of alcohol and marijuana and THC-infused alcoholic beverages.

This study had several limitations. First, testing positive for marijuana indicates marijuana use but does not necessarily imply marijuana-induced impairment at the time of crash. Given that marijuana metabolites stay longer in the urine compared to blood (AIT Laboratories 2011), we restricted the analysis to toxicological tests based on blood specimens only. Second, drug testing data are available for only about $40 \%$ of drivers involved in fatal crashes and drug testing and recording procedures may differ across states and jurisdictions (Berning and Smither 2014). Drivers with missing or incomplete toxicological testing data were excluded from our analysis. Although this data limitation may pose a threat to the external generalizability of our findings, the study design should help ensure a reasonably high level of internal validity of the results as initiators and noninitiators were paired-matched on weather, road condition, location, time of crash, traffic regulation, toxicological testing protocol, and other tempo-spatial factors. It is noteworthy that, despite the control for tempo-spatial factors through pair-matching, the study results may still be susceptible to biases from unmeasured confounders on the individual level, such as socioeconomic status, chronic substance use behavior, and comorbidities. Finally, the FARS does not record THC concentrations for 
drivers who tested positive for marijuana and thus we are unable to assess the dose-response effect of marijuana on crash initiation.

\section{Conclusions}

Results of this study indicate that marijuana use and alcohol use are each associated with a significantly increased odds of fatal 2-vehicle crash initiation. When used together, marijuana and alcohol confer a positive additive interaction effect on the odds of fatal 2-vehicle crash initiation. The decomposition analysis shows that over two-thirds of the total effect of marijuana on crash initiation are due to the direct effect whereas the remaining is due to the indirect effect through alcohol. Given the increasing prevalence of marijuana use and concurrent use of marijuana and alcohol in the driver population, multifaceted intervention programs are needed to target driving under the influence of specific substances and driving under the influence of polysubstances.

\section{Abbreviations}

AP: Attributable proportion due to interaction; BAC: Blood alcohol concentration; Cl: Confidence interval; DWI: Driving while intoxicated; FARS: Fatality Analysis Reporting System; OR: Odds ratio; RERI: Relative excess risk due to interaction; S: The synergy index; THC: Delta-9tetrahydrocannabinol

\section{Acknowledgements}

Not applicable.

\section{Role of the funder/sponsor}

The Centers for Disease Control and Prevention had no role in the preparation, review and approval of the manuscript, and the decision to submit the manuscript. Its contents are solely the responsibility of the authors and do not represent the official view of the Centers for Disease Control and Prevention.

\section{Authors' contributions}

Both authors contributed sufficiently to this manuscript; SC performed the computer programming, data management, and statistical analysis and wrote the first draft of the manuscript; and GL designed the study, secured the funding, supervised the statistical analysis, interpreted the results, and oversaw the development of the manuscript, including drafting and critical revisions. The authors read and approved the final manuscript.

\section{Funding}

This research was supported in part by the National Center for Injury Prevention and Control, Centers for Disease Control and Prevention (grant 1 R49 CE002096).

\section{Availability of data and materials}

Data from this study came from the Fatality Analysis Reporting System (FARS), maintained by the National Highway Traffic Safety Administration (NHTSA). These data are publically available and may be downloaded from https://www.nhtsa.gov/research-data/fatality-analysis-reporting-system-fars.

\section{Ethics approval and consent to participate}

This study does not meet the criteria of human subjects research per 45 CFR 46.

\section{Consent for publication}

Not application.

\section{Competing interests}

GL is Editor-in-Chief of Injury Epidemiology. He was not involved in the peerreview or handling of the manuscript. The authors have no other competing interests to disclose.

Received: 14 April 2020 Accepted: 19 August 2020

Published online: 14 September 2020

\section{References}

Asbridge M, Hayden JA, Cartwright JL. Acute cannabis consumption and motor vehicle collision risk: systematic review of observational studies and metaanalysis. BMJ. 2012;344:e536.

Bean CG, Pingel R, Hallqvist J, Berg N, Hammarström A. Poor peer relations in adolescence, social support in early adulthood, and depressive symptoms in later adulthood-evaluating mediation and interaction using four-way decomposition analysis. Ann Epidemiol. 2019;29:52-9.

Bellavia A, Valeri L. Decomposition of the Total effect in the presence of multiple mediators and interactions. Am J Epidemiol. 2018;187(6):1311-8.

Berning A, Compton R, Wochinger K. Results of the 2013-14 National Roadside Survey of Alcohol and Drug Use by Drivers. Washington, DC: National Highway Traffic Safety Administration; 2015. Report No.: DOT HS 812118.

Berning A, Smither DD. Understanding the limitations of drug test information, reporting, and testing practices in fatal crashes. Washington, DC: National Highway Traffic Safety Administration; 2014. Report No.: DOT HS 812072.

Blot WJ, Day NE. Synergism and interaction. Am J Epidemiol. 1979;110:99-100.

Blower $\mathrm{D}$. The relative contribution of truck drivers and passenger vehicle drivers to truck-passenger vehicle traffic crashes. Washington, DC: US Department of Transportation; 1998. (UMTRI-98-25).

Bonar EE, Arterberry BJ, Davis AK, Cunningham RM, Blow FC, Collins RL, et al. Prevalence and motives for drugged driving among emerging adults presenting to an emergency department. Addict Behav. 2018;78:80-4.

Center for Behavioral Health Statistics and Quality (CBHSQ). 2017 National Survey on drug use and Health: detailed tables. Rockville: Substance Abuse and Mental Health Services Administration; 2018.

Centers for Disease Control and Prevention (CDC). Death Investigation Systems. Atlanta: Centers for Disease Control and Prevention; 2015.

Chihuri S, Li G. State marijuana laws and opioid overdose mortality. Inj Epidemiol. 2019;6:38.

Chihuri S, Li G, Chen Q. Interaction of marijuana and alcohol on fatal motor vehicle crash risk: a case-control study. Inj Epidemiol. 2017;4:8.

Crancer A Jr, Dille JM, Delay JC, Wallace JE, Haykin MD. Comparison of the effects of marijuana and alcohol on simulated driving performance. Science. 1969; 164(3881):851-4.

Davis G, Hanzlick R, Denton S. The Medical Examiner and Coroner Systems: Medscape; 2015. https://emedicine.medscape.com/article/1785357overview\#a3. Accessed 21 Jan 2020.

Downey LA, King R, Papafotiou K, Swann P, Ogden E, Boorman M, et al. The effects of cannabis and alcohol on simulated driving: influences of dose and experience. Accid Anal Prev. 2013;50:879-86.

Drummer OH, Gerostamoulos J, Batziris H, Chu M, Caplehorn J, Robertson MD, et al. The involvement of drugs in drivers of motor vehicles killed in Australian road traffic crashes. Accid Anal Prev. 2004;36(2):239-48.

Dubois S, Mullen N, Weaver B, Bedard M. The combined effects of alcohol and cannabis on driving: impact on crash risk. Forensic Sci Int. 2015;248:94-100.

Executive Office of the President, National Science and Technology Council (EOPNSTC). Strengthening the Medicolegal-Death-Investigation System: Improving Data Systems. Washington, DC: Office of Science and Technology Policy; 2016.

Federal Bureau of Investigations (FBI). 2018 Crime in the United States. Clarksburg: Criminal Justice Information Services Division; 2019. https://ucr.fbi. gov/crime-in-the-u.s/2018/crime-in-the-u.s.-2018/topic-pages/tables/table-29. Accessed 21 Jan 2020.

Gjerde H, Normann PT, Christophersen AS, Samuelsen SO, Mørland J. Alcohol, psychoactive drugs and fatal road traffic accidents in Norway: a case-control study. Accid Anal Prev. 2011;43(3):1197-203.

Governors Highway Safety Association (GHSA). Drug-Impaired Driving: Marijuana and Opioids Raise Critical Issues for States. Washington, DC: Govenors Highway Safety Association; 2018.

Hartman RL, Brown TL, Milavetz G, Spurgin A, Pierce RS, Gorelick DA, et al. Cannabis effects on driving lateral control with and without alcohol. Drug Alcohol Depend. 2015;154:25-37. 
Hartman RL, Huestis MA. Cannabis effects on driving skills. Clin Chem. 2013;59(3): 478-92.

Kaplan J, Kraner J, Paulozi L. Alcohol and other drug use among victims of motor-vehicle crashes-West Virginia, 2004-2005. Morb Mortal Wkly Rep. 2006; 55(48):1293-6.

Laboratories AIT. Physician's reference for urine and blood testing and interpretation. Indianapolis: American Institute of Toxicology Laboratories; 2011.

Laumon B, Gadegbeku B, Martin JL, Biecheler MB, SAM Group. Cannabis intoxication and fatal road crashes in France: population based case-control study. BMJ. 2005;331(7529):1371

Lenne MG, Dietze PM, Triggs TJ, Walmsley S, Murphy B, Redman JR. The effects of cannabis and alcohol on simulated arterial driving: influences of driving experience and task demand. Accid Anal Prev. 2010;42(3):859-66.

Li G, Chihuri S, Brady JE. Role of alcohol and marijuana use in the initiation of fatal two-vehicle crashes. Ann Epidemiol. 2017;27(5):342-7.

Li L, Zhang X, Levine B, Li G, Zielke HR, Fowler DR. Trends and pattern of drug abuse deaths in Maryland teenagers. J Forensic Sci. 2011;56(4):1029-33.

Li MC, Brady JE, DiMaggio CJ, Lusardi AR, Tzong KY, Li G. Marijuana use and motor vehicle crashes. Epidemiol Rev. 2012;34:65-72.

Lipari RN, Hughes A, Bose J. Driving under the influence of alcohol and illicit drugs. Rockville: Center for Behavioral Health Statistics and Quality, Substance Abuse and Mental Health Services Administration; 2016.

Luedtke AR, Van der Laan MJ. Optimal individualized treatments in resourcelimited settings. Int J Biostat. 2016;12:283-303.

Moskowitz H, Hulbert S, McGlothlin WH. Marijuana effects on simulated driving performance. Accid Anal Prev. 1976;8:45-50.

National Academies of Sciences, Engineering, and Medicine (NASEM). The Health Effects of Cannabis and Cannabinoids. Washington, DC: The National Academies Press; 2017.

National Center for Statistics and Analysis (NCSA). Traffic Safety Facts 2016 Data: Alcohol Impaired Driving. Washington, DC: National Highway Traffic Safety Administration; 2017. (DOT HS 812 450).

National Center for Statistics and Analysis (NCSA). 2018 Fatal Motor Vehicle Crashes: Overview. Washington, D.C: National Highway Traffic Safety Adminstration; 2019a. (DOT HS 812 826).

National Center for Statistics and Analysis (NCSA). Fatality Analysis Reporting System (FARS) Analytic User's Manual 1975-2018. Washington, D.C: Nationa Highway Traffic Safety Adminstration; 2019b. (DOT HS 812 827).

National Conference of State Legislatures (NCSL). Marijuana Overview. Washington, D.C: National Conference of State Legislatures; 2019a. http:// www.ncsl.org/research/civil-and-criminal-justice/marijuana-overview.aspx. Accessed 21 Jan 2020.

National Conference of State Legislatures (NCSL). State Medical Marijuana Laws. Washington, DC: National Conference of State Legislatures; 2019b. http:// www.ncsl.org/research/health/state-medical-marijuana-laws.aspx. Accessed 21 Jan 2020.

National Conference of State Legislatures (NCSL). Drugged Driving: MarijuanaImpaired Driving. Washington, DC: National Conference of State Legislatures; 2019c. http://www.ncsl.org/research/transportation/drugged-drivingoverview.aspx. Accessed 21 Jan 2020.

National Highway Traffic Safety Administration (NHTSA). 2017 FARS/CRSS Coding and Validation Manual. Washington, DC: National Highway Traffic Safety Administration; 2018. (DOT HS 812 559).

Quinton S. Where doctors can recommend marijuana to replace opioids. Stateline August 13, 2019. https://www.pewtrusts.org/en/research-andanalysis/blogs/stateline/2019/08/13/where-doctors-can-recommendmarijuana-to-replace-opioids. Accessed 21 Jan 2020.

Ramaekers JG. Antidepressants and driver impairment: empirical evidence from a standard on-the-road test. J Clin Pyschiatry. 2003;64(1):20-9.

Ramaekers JG, Berghaus G, van Laar M, Drummer OH. Dose related risk of motor vehicle crashes after cannabis use. Drug Alcohol Depend. 2004;73(2):109-19.

Richiardi L, Bellocco R, Zugna D. Mediation analysis in epidemiology: methods, interpretation and bias. Int J Epidemiol. 2013;42(5):1511-9.

Robbe H. Marijuana's impairing effects on driving are moderate when taken alone but severe when combined with alcohol. Hum Psychopharmacol Clin Exp. 1998;13:S70-8.

Rogeberg O, Elvik R. The effects of cannabis intoxication on motor vehicle collision revisited and revised. Addiction. 2016;111(8):1348-59.

Rothman KJ, Greenland S, Lash TL. Modern Epidemiology. 3rd ed. Philadelphia: Lippincott, Williams, and Wilkins; 2008.
Rothman KJ, Greenland S, Walker AM. Concepts in interaction. Am J Epidemiol. 1980;112:467-70

Saracci R. Interaction and synergism. Am J Epidemiol. 1980;112:465-6.

Sewell RA, Poling J, Sofuoglu M. The effect of Cannabis compared with alcohol on driving. Am J Addict. 2009;18(3):185-93.

Smiley AM, Moskowitz H, Ziedman K. Driving simulator studies of marijuana alone and in combination with alcohol [abstract]. San Francisco, October 13: Presented at the 25th Annual Conference of the American Association for Automotive Medicine; 1981.

Substance Abuse and Mental Health Services Administration (SAMHSA). Key Substance Use and Mental Health Indicators in the United States: Results from the 2017 National Survey on Drug Use and Health. Rockville: Center for Behavioral Health Statistics and Quality, Substance Abuse and Mental Health Services Administration; 2018. (SMA 18-5068).

VanderWeele TJ. A unification of mediation and interaction: a 4-way decomposition. Epidemiology. 2014;25(5):749-61.

VanderWeele TJ. Explanation in causal inference: methods for mediation and interaction. New York: Oxford University Press; 2015.

VanderWeele TJ. The interaction continuum. Epidemiol. 2019;30(5):648-58.

Wang A, Arah OA. G-computation demonstration in causal mediation analysis. Eur J Epidemiol. 2015;30(10):1119-27.

Wong K, Brady JE, Li G. Establishing legal limits for driving under the influence of marijuana. Inj Epidemiol. 2014;1(1):26.

Zou GY. On the estimation of additive interaction by use of the four-by-two table and beyond. Am J Epidemiol. 2008;168(2):212-24.

\section{Publisher's Note}

Springer Nature remains neutral with regard to jurisdictional claims in published maps and institutional affiliations.
Ready to submit your research? Choose BMC and benefit from:

- fast, convenient online submission

- thorough peer review by experienced researchers in your field

- rapid publication on acceptance

- support for research data, including large and complex data types

- gold Open Access which fosters wider collaboration and increased citations

- maximum visibility for your research: over $100 \mathrm{M}$ website views per year

At BMC, research is always in progress.

Learn more biomedcentral.com/submissions 